\title{
Angiopoietins as Potential Targets in Management of Retinal Disease
}

\author{
Arshad M Khanani ${ }^{1,2}$ \\ Matthew W Russell ${ }^{3,4}$ \\ Aamir A Aziz ${ }^{1,2}$ \\ Carl J Danzig ${ }^{5,6}$ \\ Christina $Y$ Weng $^{7}$ \\ David A Eichenbaum ${ }^{8,9}$ \\ Rishi P Singh ${ }^{3,4}$ \\ 'Sierra Eye Associates, Reno, NV, USA; \\ ${ }^{2}$ The University of Nevada, Reno School \\ of Medicine, Reno, NV, USA; ${ }^{3}$ Center for \\ Ophthalmic Bioinformatics, Cole Eye \\ Institute, Cleveland Clinic, Cleveland, \\ $\mathrm{OH}$, USA; ${ }^{4}$ Cleveland Clinic Lerner \\ College of Medicine, Case Western \\ Reserve University, Cleveland, OH, USA; \\ ${ }^{5}$ Rand Eye Institute, Deerfield, FLA, USA; \\ ${ }^{6}$ Florida Atlantic University, Charles E. \\ Schmidt College of Medicine, Boca \\ Raton, FL, USA; ${ }^{7}$ Baylor College of \\ Medicine, Houston, TX, USA; ${ }^{8}$ Retina \\ Vitreous Associates of Florida, St \\ Petersburg, FLA, USA; ' University of \\ South Florida Morsani College of \\ Medicine, Tampa, FLA, USA
}

Correspondence: Arshad M Khanani 950 Ryland Street, Reno, NV, 89502, USA Tel +775 329-0286

Fax +775-329-0849

Email arshad.khanani@gmail.com

\begin{abstract}
The Ang/Tie2 pathway complements VEGF-mediated activity in retinal vascular diseases such as DME, AMD, and RVO by decreasing vascular integrity, increasing neovascularization, and increasing inflammatory signaling. Faricimab is a bispecific antibody that has been developed as an inhibitor of both VEGF and Ang2 that has shown positive results in phase I, II and III trials. Recent Year 1 data from phase III clinical trials YOSEMITE, RHINE, TENAYA, and LUCERNE have confirmed the efficacy, safety, durability, and superiority of faricimab in patients with DME and nAMD. Faricimab, if approved, may significantly decrease treatment burden in patients with retinal vascular diseases to a greater extent than would current standard of care anti-VEGF injections.
\end{abstract}

Keywords: Ang/Tie, faricimab, YOSEMITE, RHINE, TENAYA, LUCERNE

\section{Introduction}

Diabetic retinopathy (DR), diabetic macular edema (DME), and neovascular agerelated macular degeneration (nAMD), are leading causes of ocular morbidity worldwide. ${ }^{1}$ With the increasing aging population and a rapidly growing prevalence of diabetes mellitus globally, the demand for treatment is expected to continuously rise. For example, prevalence of diabetes mellitus in the United States is predicted to increase by $165 \%$ through 2050 with ocular manifestations occurring in over $60 \%$ of these patients. ${ }^{2,3}$

The common pathogenesis of these retinal vascular diseases involves increased production of vascular endothelial growth factor (VEGF), a pro-angiogenic molecule. The VEGF protein family is comprised of VEGF-A, VEGF-B, VEGF-C, and VEGF-D. ${ }^{4}$ VEGF-A is of high interest due to its pro-angiogenic effects and ability to increase vascular permeability. ${ }^{5,6}$ Current standard of care for nAMD and visually significant DME is intravitreal injection of VEGF inhibitors. Both FDAapproved and off-label anti-VEGF drugs are currently used although not all are approved for all indications: aflibercept (Eylea, FDA-approved), ranibizumab (Lucentis, FDA-approved), bevacizumab (Avastin, off-label), and brolucizumab (Beovu, not approved for DME, FDA-approved for neovascular AMD).

While anti-VEGF injections are generally quite effective in the management of these retinal conditions, they often require a high frequency of treatment, placing a heavy burden on patients, providers, and society. The need for constant clinic visits and injections has contributed to lapses in care, which may affect as many as $46 \%$ of DME patients, attributable to reasons including long travel time to hospitals and dissatisfaction with benefits. ${ }^{7-9}$ These limitations highlight the crucial need to 
identify novel treatment mechanisms of retinal disease that can improve efficacy and reduce injection frequency, thereby allowing for optimization of visual outcomes and reduction of treatment burden.

One complementary pathway that has been recently explored as a potential target for novel medications is the angiopoetin-Tie2 (Ang/Tie2) axis which helps regulate vascular permeability and inflammation. This review summarizes recent evidence regarding the function of angiopoietins and their role as a pharmacologic target in the angiopoietin-Tie 2 cascade, with a focus on clinical trial data evaluating the agent faricimab in the management of retinal vascular diseases.

\section{Materials and Methods}

This review was performed with the primary objective of this study to summarize available literature regarding the Ang/Tie2 pathway, and outcomes of therapeutics designed to modulate this pathway for clinical benefit in patients with retinal vascular diseases. Preclinical studies, case reports, case series, observational studies, and randomized controlled trials were considered for inclusion.

The National Clinical Trial (NCT) database was queried for the following terms: "(Diabetic Macular Edema) OR (Diabetic Retinopathy) OR (Age Related Macular Degeneration) alongside (Faricimab) OR (Angiopoietin), OR (Ang2) OR (Ang/Tie). PubMed was queried for the following terms:

(Age-Related Macular Degeneration) OR (exudative AMD) OR (neovascular AMD) OR (nonexudative AMD) OR (dry AMD) OR (diabetic macular edema) OR (diabetic retinopathy)) AND ((Faricimab) OR (Ang2) OR (Angiopoietin) OR. (Ang/tie)))

Additional articles were identified from a manual search of reference lists within included articles. The full text of each article was reviewed by MWR.

\section{Discussion}

\section{Basic and Translational Science of the Ang/Tie2 Pathway \\ Key Signaling Components}

While the VEGF pathway is involved in certain stages of angiogenesis including controlling vascular permeability, the Ang/Tie2 pathway is also involved in distinct and important stages of angiogenesis such as vessel remodeling and maturation. ${ }^{10}$ The Ang/Tie2 axis uses tyrosine kinase signaling and has key components including the Tie-1 and Tie-2 tyrosine kinase receptors with both intra and extracellular domains. ${ }^{11}$ Ang-1, Ang-2, and Ang-4 are all secreted ligands that bind to the Tie2 receptor. ${ }^{12}$ Tie-1 does not directly bind to Ang proteins, but does assist in signal transduction by forming complexes with Tie2. ${ }^{13}$ Following physiologic activation and ligand-receptor complex formation with constitutively secreted Ang-1 in homeostasis, Tie2 becomes phosphorylated, leading to downstream activation of cellular pathways AKT and ERK, which are involved in decreasing angiogenesis and vascular permeability, favoring vascular stability (Figure 1). ${ }^{10}$ Ang-1 and Ang-4 are known agonists of the Tie-2 receptor and are bound to Tie 2 in the physiologic, homeostatic state. ${ }^{14,15}$ In pathophysiologic states, Ang-2 is highly secreted and more plentiful than Ang-1. By acting as a competitive inhibitor of Ang-1 and Ang-4 binding, Ang-2 functions as a negative regulator, which leads to dephosphorylation of Tie2. 10,16,17 Furthermore, vascular endothelial tyrosine phosphatase (VE-PTP), is another negative regulator of Tie 2 signaling. VE-PTP achieves this through dephosphorylation of Tie-2. Therefore, decreasing activity of both Ang-2 and VE-PTP could also potentially increase physiologic Tie-2 signaling; thus, both Ang-2 and VE-PTP are drug targets of interest. $^{18-20}$

\section{Tie2 and Inflammation}

Increased presence of pro-inflammatory cytokines is another common feature in DR, AMD, and RVO. ${ }^{21-23}$ Inflammatory stimuli trigger release of Ang-2 from Weibel-Palade bodies of endothelial cells, leading to the disruptive effects of abundant Ang-2. The subsequent dephosphorylation of the Tie-2 receptor leads to downstream pro-inflammatory signaling that results in decreased vascular integrity. ${ }^{16}$ Physiologic Tie2 activation may also suppress NF-kB, reducing intracellular inflammatory signaling, while enhancing the anti-inflammatory cascade of Tie 2 signaling. ${ }^{24}$

\section{Tie2 and Vascular Permeability}

Multiple pathways downstream of Ang/Tie2 affect vascular permeability. Healthy vasculature and intact tight endothelial junctions are key to normal retinal physiology. Protein kinase $\mathrm{B}$ activated by physiologic Ang/Tie2 signaling has been shown to enhance endothelial cell survival, an essential component of vascular integrity. ${ }^{25,26}$ Activation of phosphatidylinositol 3-kinase pathway can enhance endothelial cell 


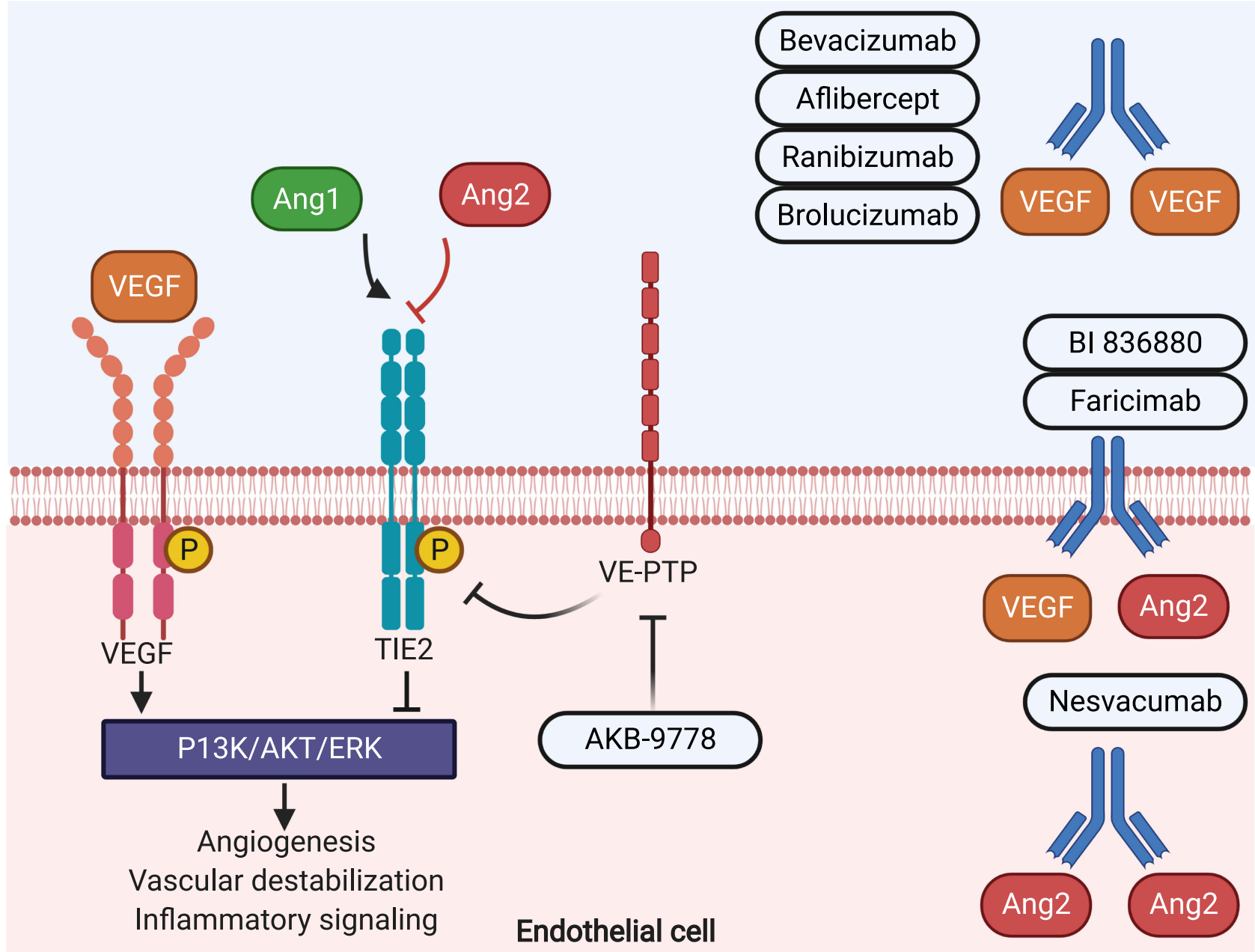

Figure I Ang/Tie2 axis: Ang2 competitively inhibits binding of Angl to Tie2 receptor. When bound to Angl, Tie2 autophosphorylates and enhances vascular stability through PI3K, AKT, and ERK signaling. VE-PTP dephosphorylates Tie2. VEGF binding and autophosphorylation further leads to increased angiogenesis. Faricimab and BI 836880 are bispecific antibodies containing domains for binding VEGF and Ang2. Nesvacumab is an antibody with binding domains for Ang2. AKB-9778 is an inhibitor of VEPTP. Created withBioRender.com.

migration. ${ }^{27}$ Physiologic Ang/Tie2 signaling has been shown to tighten endothelial cell junctions, through organization of vascular endothelial cell cadherin complexes. ${ }^{28}$ Ang/Tie2 signaling can also lead to downstream inactivation of transcription factor FOXO1, which decreases Ang-2 production, thus decreasing levels of this disruptive pathologic regulator. ${ }^{29}$

In animal models, activation of Ang/Tie2 signaling has been shown to reduce pulmonary vascular edema, vascular stroke size, and renal edema. ${ }^{30-32}$ This animal data imply that this pathway could be relevant in human disease states including DME and AMD, where restoring vascular integrity is essential. In porcine retinal endothelial cells, it has been found that in the presence of Ang-2, VEGF-A-induced vascular permeability increases threefold, suggesting these pathways are complementary, or

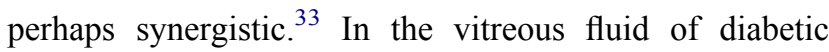

human eyes, it has been shown that Ang-2 levels are markedly elevated compared to controls and correlate with biomarkers of poor prognosis. ${ }^{34,35}$ These findings are expected since environmental factors, such as hypoxia, oxidative stress, and hypertriglyceridemia, that are present in diabetic patients have been shown to increase levels of Ang-2. ${ }^{36-39}$ This imbalance in signaling in diabetic patients burdened with excessive Ang-2 implies that Ang-2 may be a key driver in decreasing vascular integrity in diabetic eyes. The combination of hyperglycemia, hypoxia, and increased inflammatory factors in diabetics may serve as an angiogenic switch leading to an increase in Ang2 secretion.

\section{Tie2 and Neovascularization}

Neovascularization is heavily involved in pathogenesis of retinovascular disorders. It was found that SNPs (single 
nucleotide polymorphisms) in the gene that encode for angiopoietin-2 (ANGPT2) resulted in increased susceptibility for developing nAMD. ${ }^{40}$ Similar to the findings in diabetic human eyes, vitreous samples of human eyes with nAMD demonstrate elevation of Ang-2 in both disease states when compared to controls. ${ }^{41,42}$

\section{Clinical Studies Targeting the Ang/Tie2 Pathway AKB-9778}

Informed on the basic science work above, the Ang/Tie2 pathway became an area of active investigation. One molecule involving Tie2 regulation was AKB-9778, developed by Aerpio Pharmaceuticals. AKB-9778 is a smallmolecule inhibitor of VE-PTP, which downstream results in increased Tie2 signaling and subsequent vascular stability. AKB-9778 is administered as a subcutaneous injection, similar to diabetic patients currently administering insulin. It was most recently evaluated as an ocular therapeutic in the TIME-2b clinical trial, where Nonproliferative Diabetic Retinopathy (NPDR) subjects received it as a monotherapy to evaluate reductions in the number of patients with worsening Diabetic Retinopathy Severity Scale (DRSS) scores.

\section{TIME-2b}

TIME-2b trial was a Phase II trial, which tested the efficacy of AKB-9778 (an inhibitor of VE-PTP) in 167 patients with NPDR. In this trial, patients were assigned to subcutaneous AKB-9778 15mg once daily (and one subcutaneous placebo daily), twice daily, or two subcutaneous placebo treatments daily. This trial did not meet its primary endpoint, as the effect on the DRSS score was the same at 48 weeks as it was at 12 weeks and no improvements were seen. No reduction in the percentage of patients progressing to visually threatening diabetic retinopathy was demonstrated. However, reduction in intraocular pressure (IOP) and improvement to the urine albuminto-creatinine ratio did demonstrate Tie2 activation, implying that the AKB-9778 dose may need to be increased' or combined with a ranibizumab injection for ocular efficacy. $^{12,43}$ AKB-9778 is now being investigated for improvements in diabetic nephropathy.

\section{Nesvacumab}

Nesvacumab, developed by Regeneron, is an immunoglobulin that selectively binds Ang2 with high affinity, thus preventing Ang2/Tie2 receptor binding and complex formation. Nesvacumab is administered through intravitreal injection and is given in combination with aflibercept. ${ }^{44}$ In a Phase I dose escalation trial, 20 patients with AMD or DME were divided into the following arms (2 patients with AMD and 2 with DME per arm: $0.5 \mathrm{mg}$ nesvacumab $+2 \mathrm{mg}$ aflibercept, $1 \mathrm{mg}$ nesvacumab $+2 \mathrm{mg}$ aflibercept, $3 \mathrm{mg}$ nesvacumab $+2 \mathrm{mg}$ aflibercept, $6 \mathrm{mg}$ nesvacumab $+2 \mathrm{mg}$ aflibercept, or $6 \mathrm{mg}$ nesvacumab alone. Visual and anatomic improvements were noted in all doses, prompting further phase II investigation through the ONYX and RUBY trials. ${ }^{45}$

\section{ONYX and RUBY}

The ONYX and RUBY trials were two randomized, double masked, controlled phase II studies of nesvacumab and aflibercept coformulation in patients with nAMD and DME, respectively. In these trials, patients were divided into three arms: $3 \mathrm{mg}$ nesvacumab $+2 \mathrm{mg}$ aflibercept, $6 \mathrm{mg}$ nesvacumab $+2 \mathrm{mg}$ aflibercept, or $2 \mathrm{mg}$ aflibercept. Patients were dosed every four weeks (Q4W) for 12 weeks. Primary endpoints for the study were changes in best corrected visual acuity (BCVA) from weeks 12 and 36. No significant differences were noted in BCVA or central subfield thickness (CST) for either study. However, in the higher dose formulation of the Ruby trial (6mg nesvacumab $+2 \mathrm{mg}$ aflibercept), a significant proportion of patients had $\mathrm{a} \geq 2$-step improvement in the DRSS score from baseline to week 12, compared to aflibercept-alone patients. Due to these findings, nesvacumab did not warrant phase III investigation. ${ }^{46,47}$

\section{Faricimab}

Faricimab, developed by Roche, was a biologic treatment developed to target both VEGF and Ang2. Faricimab is a bispecific antibody that binds both VEGF-A and Ang2 without inhibition of Ang1. ${ }^{48-50}$ Through inhibition of both ligands, it was thought that faricimab could result in improved retinal outcomes and decreased treatment frequency in patients with DME, nAMD, and RVO.

The first Phase I clinical trial for faricimab was conducted in 12 nAMD patients. Patients were placed in $0.5 \mathrm{mg}, 1.5 \mathrm{mg}, 3 \mathrm{mg}$ and $6 \mathrm{mg}$ dose-escalating arms. Furthermore, 6 patients were also given three treatments of $3 \mathrm{mg}$ and $6 \mathrm{mg}$ faricimab over an 8 -week interval. It was found that faricimab exhibited a favorable safety profile with evidence of improvements in BCVA and disease state as seen on OCT imaging, with no dose-limiting toxicities in any group. ${ }^{51}$ Following this success, two Phase II 
studies were conducted to study the efficacy and safety of faricimab in nAMD (AVENUE \& STAIRWAY), and one trial in DME (BOULEVARD). These studies compared the efficacy of these treatments to standard-of-care ranibizumab, an anti-VEGF therapy.

\section{AVENUE}

This Phase II, randomized, comparator-controlled, double-masked, 273-patient trial evaluated the safety, efficacy, and dosing of faricimab in treatment-naïve patients with nAMD. This 36-week study divided patients $3: 2: 2: 2: 3$ into five arms. The first arm was a group of control patients who were given ranibizumab $0.5 \mathrm{mg}$ Q4W. Arms involving faricimab treatments were $1.5 \mathrm{mg}$ Q4W, 6mg Q4W, and 6mg every 8 weeks (Q8W). One final group was also given $0.5 \mathrm{mg}$ ranibizumab $\mathrm{Q} 4 \mathrm{~W}$ for 8 weeks, followed by faricimab $6 \mathrm{mg}$ Q4W. The primary endpoint for this trial evaluated mean change in BCVA from baseline to Week 36, with secondary endpoints evaluating anatomic parameters and safety signals. All groups in this trial demonstrated improvement in BCVA and reduction in CST. Although faricimab did not meet its primary endpoint of superiority to Q4W ranibizumab at week 36, the agent was not significantly inferior to ranibizumab with respect to changes in BCVA. Visual and anatomical improvements were demonstrated in faricimab arms, while no signs of safety concerns were noted. $^{52}$

\section{STAIRWAY}

The STAIRWAY study was a 76-patient, double-masked, randomized, comparator-controlled, Phase II trial to evaluate the safety, efficacy, and dosing of faricimab in treatment-naïve patients with nAMD. This study also explored the extended faricimab dosing compared with monthly dosing in this patient population. Patients were randomized 1:1:1 to either ranibizumab $0.5 \mathrm{mg}$ Q4W as a control, faricimab $6 \mathrm{mg}$ every 12 weeks (Q12W), or faricimab $6 \mathrm{mg}$ every 16 weeks $(\mathrm{Q} 16 \mathrm{~W})$. The primary endpoint for this trial evaluated mean change in BCVA from baseline to Week 40, while secondary endpoints again evaluated changes in anatomic parameters, the percentage of patients maintaining extended dosing intervals, and safety signals. After 52 weeks of treatment, Q12W and Q16W faricimab groups demonstrated comparable results to ranibizumab with respect to BCVA and CST. Patients in the Q16W arm who demonstrated disease activity 12 weeks after the four loading doses were continued at a Q12W dosing interval, rather than extended up to Q16W dosing. However, 65\% of the patients in both faricimab groups showed no disease activity at week 24 . Over 52 weeks, BCVA improvements were as follows: Q16W faricimab (+11.4 letters), Q12W faricimab (+10.1 letters) and Q4W ranibizumab (+9.6 letters). All three groups showed comparable CST reductions and no safety signals were observed. This study demonstrated that faricimab was not only efficacious but also has the potential to reduce treatment frequency in this patient population as results in the extended treatment regimens were comparable to a monthly dose of ranibizumab. ${ }^{53}$

\section{BOULEVARD}

The BOULEVARD study was another comparatorcontrolled, double-masked Phase II randomized clinical trial with 229 patients that studied faricimab in treatment of center-involving DME, with both treatment-naïve patients and patients previously treated with anti-VEGF injections, over a 36-week period. Treatment-naïve patients were randomized 1:1:1 into three arms, where the control arm received ranibizumab $0.3 \mathrm{mg} \mathrm{Q} 4 \mathrm{~W}$ while the faricimab groups were given either $1.5 \mathrm{mg}$ or $6.0 \mathrm{mg}$ dosed Q4W. Previously treated patients were randomized $1: 1$ in either ranibizumab $0.3 \mathrm{mg}$ Q4W and faricimab $6.0 \mathrm{mg}$ Q4W. All subjects received intravitreal injections Q4 weeks through week 20. This was followed by an offtreatment period, which could last up to week 36, the final study visit. The primary endpoint for this trial evaluated the mean change in BCVA from baseline to Week 24, while secondary endpoints included evaluation of anatomic parameters and safety signals. This study met its primary endpoint at 24 weeks, where the $6.0 \mathrm{mg}$ dose of faricimab arm had statistically significant improvements in BCVA when compared to other treatment groups. Patients in the faricimab group were also noted to experience reductions in CST, and DRSS score improvement compared to ranibizumab. ${ }^{54}$

Due to success of the AVENUE, STAIRWAY, and BOULEVARD trials, faricimab was identified as a candidate warranting further investigation. Global Phase III studies were initiated to evaluate long-term safety, durability, and efficacy of faricimab for treatment of AMD and DME. In these trials, aflibercept was used as standard of care treatment, after it was shown to increase likelihood of visual improvement in patients with DME. $^{55}$ 


\section{TENAYA and LUCERNE}

TENAYA and LUCERNE trials were two identical global, randomized, double masked, active-comparator Phase III trials that evaluated the safety and efficacy of faricimab compared to aflibercept in patients with nAMD. The TENAYA trial studied 671 patients with nAMD while the LUCERNE trial enrolled 658 patients. The $6 \mathrm{mg}$ faricimab arms started with an initial four Q4W loading doses, followed by dosing intervals up to Q16W, which can be dropped down to $\mathrm{Q} 12 \mathrm{~W}$ or $\mathrm{Q} 8 \mathrm{~W}$ after disease activity assessments done at pre-specified timepoints. The control arm was aflibercept at Q8W. Sham injections were also administered at study appointments to maintain masking between arms.

The primary endpoint of these two studies was mean BCVA change from baseline at Week 48 as an average of Weeks 40, 44, and 48. New data for both trials was released in Q1 of 2021, showing positive results for faricimab. In the Year 1 TENAYA trial results, average visual gains were +5.8 letters in faricimab groups and +5.1 letters in aflibercept group. In the Year 1 LUCERNE trial results, average visual gains were +6.6 letters in both experimental and control groups. In faricimab treated patients, $80 \%$ of the patients were able to be given doses at Q12W intervals or longer. Faricimab-treated patients being dosed Q16W also experienced similar reductions in CST compared to aflibercept-treated patients. The TENAYA and LUCERNE trial results suggest that faricimab could reduce treatment frequency and improve visual outcomes in patients with nAMD. $^{58,59}$

\section{YOSEMITE and RHINE}

YOSEMITE and RHINE trials were two identical global, randomized, double masked, active-comparator Phase III trials that evaluated the safety and efficacy of faricimab compared to aflibercept in patients with DME. The YOSEMITE trial studied 940 subjects, while the RHINE trial enrolled 951 subjects. Both treatmentnaïve and previously treated patients were randomized 1:1:1 into three treatment arms: faricimab $6 \mathrm{mg}$ at personalized dosing intervals up to Q16W, faricimab $6 \mathrm{mg}$ Q12W, or aflibercept Q8W. Subjects in the personalized dosing interval arm could have their treatment interval shortened based on disease activity assessments. Sham injections were also administered at study appointments where no injection was scheduled to maintain masking between arms. The primary endpoint of this study was mean change in BCVA at 52 weeks. In the Year 1
YOSEMITE trial results, the average vision gains were $+11.6,+10.7$, and +10.9 letters in the faricimab personal dosing interval, Q8W faricimab arm, and aflibercept arm respectively. In the Year 1 RHINE trial results, the average vision gains were $+10.8,+11.8$, and +10.3 letters in the faricimab personal dosing interval, Q8W faricimab arm, and aflibercept arm, respectively.

Secondary endpoints also found that out of patients in the faricimab personalized dosing interval arm in both trials, $70 \%$ achieved dosing intervals of 3 months or longer, suggesting that treatment frequency in faricimab can be extended longer than current standard-of-care treatment regimens. Furthermore, patients who were given faricimab at intervals of up to 4 months also experienced greater reductions in CST when compared to aflibercept at two-month dosing intervals. YOSEMITE and RHINE results suggest that faricimab could reduce treatment frequency and improving visual outcomes as compared to current standard-of-care therapy. ${ }^{56,57}$ The value of a more efficacious agent is especially paramount for DME patients, who often suffer from longer lapses of care than their nAMD or RVO counterparts.

\section{BI 836880}

BI 836880 , is a new bispecific nanobody developed by Boehringer Ingelheim, which has domains for binding VEGF and Ang2 in a similar manner to faricimab, along with an albumin-binding domain for half-life extension. BI 836880 is administered via intravitreal injection. An openlabel, non-randomized, Phase I study is currently underway to evaluate the safety and tolerability of this agent. This study will contain a single rising dose segment, followed by a multiple rising dose segment. Primary endpoints focus on safety and tolerability, and will include the number of patients with dose limiting events and drug-related adverse effects. This study is expected to reach completion by March $2022 .{ }^{60}$

\section{Conclusion}

The Ang/Tie2 axis complements VEGF-mediated activity in retinovascular diseases such as DME, AMD, and RVO by decreasing vascular integrity, increasing neovascularization, and increasing inflammatory signaling. Faricimab has been developed as an inhibitor of both VEGF and Ang2. Recent Year 1 data from Phase III clinical trials suggest that faricimab is safe, efficacious, and may hold potential to reduce treatment burden in patients with nAMD and DME. Further data from these studies will need to be collected and analyzed in order to determine 
if faricimab is superior to standard of care anti-VEGF medication in the real-world management of these conditions.

\section{Disclosure}

AK: Consultant: Adverum, Aerpio, Allergan, Chengdu Kanghong, DORC, Genentech, Inc., Kato, Kodiak, Novartis, Gemini, Graybug, Gyroscope, Opthea, Oxurion, PolyPhotonix, Recens Medical, Regenxbio, Roche

Research support: Adverum, Alkahest, Allegro, Allergan, Chengdu Kanghong, Gemini, Genentech, Inc., Gyroscope, Iveric Bio, NGM, Neurotech, Kodiak, Novartis, Opthea, Oxurion, Regenxbio, Recens Medical, Roche

Lecture fees: Allergan, Genentech, Novartis

CD: Consultant: Genentech, Roche, Regeneron, Novartis, IvericBio

Research support: Genentech, Roche, Regeneron, Novartis, IvericBio, Kodiak, Adverum, Gyroscope

Lecture fees: Novartis, DORC

CYW: Consultant: Allergan/AbbVie, Alcon, Alimera

Sciences, Novartis, Regeneron, REGENXBIO, Genentech, DORC

DE: Consultant: Genentech, Regeneron, Allergan, Novartis, Notal Vision, EyePoint, Gyroscope, Kodiak, RecensMedical, DORC, IvericBio, Apellis, KKR, Regenxbio, Bausch \& Lomb

Research support: AsclepiX, Genentech, Bayer, Novartis, Alimera, Opthea, Ocular Therapeutix, EyePoint, Mylan, Chengdu, Gemini, Gyroscope, Kodiak, NGM, RecensMedical, Alkahest, Ionis, IvericBio, Regenxbio

Lecture fees: Genentech, Bayer, Allergan, Novartis, EyePoint, DORC, Apellis

Equity/Stockholder: Clearside, US Retina, Hemera Biopharmaceuticals, Boston Image Reading Center, Network Eye

Founder: Network Eye

RPS: Consultant: Regeneron, Genentech, Alcon, Novartis, Asclepix, Gyroscope, Bausch and Lomb.

Research support: Apellis, NGM Biopharma

The authors report no other conflicts of interest in this work.

\section{References}

1. Bourne RRA, Stevens GA, White RA, et al. Causes of vision loss worldwide, 1990-2010: a systematic analysis. Lancet Glob Health. 2013;1(6):e339-349. doi:10.1016/S2214-109X(13) 70113-X
2. Boyle JP, Honeycutt AA, Narayan KMV, et al. Projection of diabetes burden through 2050: impact of changing demography and disease prevalence in the U.S. Diabetes Care. 2001;24(11):1936-1940. doi:10.2337/diacare.24.11.1936

3. Fong DS, Aiello L, Gardner TW, et al. Retinopathy in diabetes. Diabetes Care. 2004;27(suppl 1):s84-7. doi:10.2337/diacare.27.2007.S84

4. Ferrara N, Gerber H-P, LeCouter J. The biology of VEGF and its receptors. Nat Med. 2003;9(6):669-676. doi:10.1038/nm0603-669

5. Boyer DS, Hopkins JJ, Sorof J, Ehrlich JS. Anti-vascular endothelial growth factor therapy for diabetic macular edema. Ther $A d v$ Endocrinol Metab. 2013;4(6):151-169. doi:10.1177/ 2042018813512360

6. Wang W, Lo ACY. Diabetic retinopathy: pathophysiology and treatments. Int J Mol Sci [Internet]. 2018;19(6):1816.

7. akamura Y, Ohkoshi K, Murata T. New Strategies for Treatment of Diabetic Macular Edema. Journal of Ophthalmology. 2018;2018: e4292154.

8. Weiss M, Sim DA, Herold T, et al. Compliance and adherence of patients with diabetic macular edema to intravitreal anti-vascular endothelial growth factor therapy in daily practice. Retina Phila Pa. 2018;38(12):2293-2300. doi:10.1097/IAE.0000000000001892

9. Boulanger-Scemama E, Querques G, About F, et al. Ranibizumab for exudative age-related macular degeneration: a five year study of adherence to follow-up in a real-life setting. $J \mathrm{Fr}$ Ophtalmol. 2015;38(7):620-627. doi:10.1016/j.jfo.2014.11.015

10. Thurston G, Daly C. The complex role of angiopoietin-2 in the angiopoietin-tie signaling pathway. Cold Spring Harb Perspect Med. 2012;2(9):a006650.

11. Schnürch H, Risau W. Expression of tie-2, a member of a novel family of receptor tyrosine kinases, in the endothelial cell lineage. Dev Camb Engl. 1993;119(3):957-968.

12. Whitehead M, Osborne A, Widdowson PS, Yu-Wai-Man P, Martin KR. Angiopoietins in diabetic retinopathy: current understanding and therapeutic potential. J Diabetes Res. 2019;;2019:1-9. doi:10.1155/2019/5140521

13. Joussen AM, Ricci F, Paris LP, Korn C, Quezada-Ruiz C, Zarbin M Angiopoietin/Tie2 signalling and its role in retinal and choroidal vascular diseases: a review of preclinical data. Eye. 2021;35:1-12.

14. Brunckhorst MK, Wang H, Lu R, Yu Q. Angiopoietin-4 promotes glioblastoma progression by enhancing tumor cell viability and angiogenesis. Cancer Res. 2010;70(18):7283-7293. doi:10.1158/ 0008-5472.CAN-09-4125

15. Kim W. The role of angiopoietin-1 in kidney disease. Electrolytes Blood Press E BP. 2008;6(1):22-26. doi:10.5049/EBP.2008.6.1.22

16. Scholz A, Plate KH, Reiss Y. Angiopoietin-2: a multifaceted cytokine that functions in both angiogenesis and inflammation. Ann N Y Acad Sci. 2015;1347:45-51. doi:10.1111/nyas. 12726

17. Korhonen EA, Lampinen A, Giri H, et al. Tie1 controls angiopoietin function in vascular remodeling and inflammation. $J$ Clin Invest. 2016;126(9):3495-3510. doi:10.1172/JCI84923

18. Li Z, Huang H, Boland P, et al. Embryonic stem cell tumor model reveals role of vascular endothelial receptor tyrosine phosphatase in regulating Tie2 pathway in tumor angiogenesis. Proc Natl Acad Sci U S A. 2009;106(52):22399-22404. doi:10.1073/pnas.0911189106

19. Winderlich M, Keller L, Cagna G, et al. VE-PTP controls blood vessel development by balancing Tie-2 activity. $J$ Cell Biol. 2009;185(4):657-671. doi:10.1083/jcb.200811159

20. Frye $M$, Dierkes $M$, Küppers V, et al. Interfering with VE-PTP stabilizes endothelial junctions in vivo via Tie-2 in the absence of VE-cadherin. $J$ Exp Med. 2015;212(13):2267-2287. doi:10.1084/ jem.20150718

21. Wang Y, Wang VM, Chan -C-C. The role of anti-inflammatory agents in age-related macular degeneration (AMD) treatment. Eye. 2011;25 (2):127-139. doi:10.1038/eye.2010.196 
22. Doganay S, Evereklioglu C, Er H, et al. Comparison of serum NO, TNF- $\alpha$, IL-1 $\beta$, sIL-2R, IL- 6 and IL-8 levels with grades of retinopathy in patients with diabetes mellitus. Eye. 2002;16(2):163-170. doi:10.1038/sj/eye/6700095

23. Deobhakta A, Chang LK. Inflammation in retinal vein occlusion. Int J Inflamm. 2013;2013:438412. doi:10.1155/2013/438412

24. Hughes DP, Marron MB, Brindle NPJ. The antiinflammatory endothelial tyrosine kinase Tie2 interacts with a novel nuclear factor-kappaB inhibitor ABIN-2. Circ Res. 2003;92(6):630-636. doi:10.1161/01.RES.0000063422.38690.DC

25. Papapetropoulos A, Fulton D, Mahboubi K, et al. Angiopoietin-1 inhibits endothelial cell apoptosis via the Akt/survivin pathway. J Biol Chem. 2000;275(13):9102-9105. doi:10.1074/jbc.275.13.9102

26. Kontos CD, Cha EH, York JD, Peters KG. The endothelial receptor tyrosine kinase Tie1 activates phosphatidylinositol 3-kinase and Akt to inhibit apoptosis. Mol Cell Biol. 2002;22(6):1704-1713. doi:10.1128/MCB.22.6.1704-1713.2002

27. Augustin HG, Koh GY, Thurston G, Alitalo K. Control of vascular morphogenesis and homeostasis through the angiopoietin-Tie system. Nat Rev Mol Cell Biol. 2009;10(3):165-177. doi:10.1038/nrm2639

28. Mirando AC, Shen J, Silva RLE, et al. A collagen IV-derived peptide disrupts $\alpha 5 \beta 1$ integrin and potentiates Ang2/Tie2 signaling. JCI Insight. 2019;4(4). doi:10.1172/jci.insight.122043.

29. Daly C, Wong V, Burova E, et al. Angiopoietin-1 modulates endothelial cell function and gene expression via the transcription factor FKHR (FOXO1). Genes Dev. 2004;18(9):1060-1071. doi:10.1101/ gad. 1189704

30. Dekker N, van Meurs M, van Leeuwen ALI, et al. Vasculotide, an angiopoietin-1 mimetic, reduces pulmonary vascular leakage and preserves microcirculatory perfusion during cardiopulmonary bypass in rats. $B r \quad J$ Anaesth. 2018;121(5):1041-1051. doi:10.1016/j. bja.2018.05.049

31. Gurnik S, Devraj K, Macas J, et al. Angiopoietin-2-induced blood-brain barrier compromise and increased stroke size are rescued by VE-PTPdependent restoration of Tie2 signaling. Acta Neuropathol (Berl). 2016;131(5):753-773. doi:10.1007/s00401-016-1551-3

32. Rübig E, Stypmann J, Van Slyke P, et al. The synthetic tie2 agonist peptide vasculotide protects renal vascular barrier function in experimental acute kidney injury. Sci Rep. 2016;6:22111. doi:10.1038/srep22111

33. Peters S, Cree IA, Alexander R, et al. Angiopoietin modulation of vascular endothelial growth factor: effects on retinal endothelial cell permeability. Cytokine. 2007;40(2):144-150. doi:10.1016/j. cyto.2007.09.001

34. Watanabe D, Suzuma K, Suzuma I, et al. Vitreous levels of angiopoietin 2 and vascular endothelial growth factor in patients with proliferative diabetic retinopathy. Am J Ophthalmol. 2005;139 (3):476-481. doi:10.1016/j.ajo.2004.10.004

35. Loukovaara S, Robciuc A, Holopainen JM, et al. Ang-2 upregulation correlates with increased levels of MMP-9, VEGF, EPO and TGF $\beta 1$ in diabetic eyes undergoing vitrectomy. Acta Ophthalmol (Copenh). 2013;91(6):531-539. doi:10.1111/j.1755-3768.2012.02473.x

36. Oh H, Takagi H, Suzuma K, Otani A, Matsumura M, Honda Y. Hypoxia and vascular endothelial growth factor selectively up-regulate angiopoietin-2 in bovine microvascular endothelial cells. J Biol Chem. 1999;274(22):15732-15739.

37. Ohashi H, Takagi H, Koyama S, et al. Alterations in expression of angiopoietins and the Tie-2 receptor in the retina of streptozotocin induced diabetic rats. Mol Vis. 2004;10:608-617.

38. Park SW, Yun J-H, Kim JH, Kim K-W, Cho C-H, Kim JH. Angiopoietin 2 induces pericyte apoptosis via $\alpha 3 \beta 1$ integrin signaling in diabetic retinopathy. Diabetes. 2014;63(9):3057-3068. doi:10.2337/db13-1942

39. Lee SG, Lee CG, Yun IH, Hur DY, Yang JW, Kim HW. Effect of lipoic acid on expression of angiogenic factors in diabetic rat retina. Clin Experiment Ophthalmol. 2012;40(1):e47-57. doi:10.1111/ j.1442-9071.2011.02695.x
40. Ma L, Brelen ME, Tsujikawa M, et al. Identification of ANGPT2 as a new gene for neovascular age-related macular degeneration and polypoidal choroidal vasculopathy in the Chinese and Japanese populations. Invest Ophthalmol Vis Sci. 2017;58(2):1076-1083. doi:10.1167/iovs.16-20575

41. Ng DS, Yip YW, Bakthavatsalam M, et al. Elevated angiopoietin 2 in aqueous of patients with neovascular age related macular degeneration correlates with disease severity at presentation. Sci Rep. 2017;7:45081. doi:10.1038/srep45081

42. Huber M, Wachtlin J. Vitreous levels of proteins implicated in angiogenesis are modulated in patients with retinal or choroidal neovascularization. Ophthalmol $J$ Int Ophtalmol Int $J$ Ophthalmol $Z$ Augenheilkd. 2012;228(3):188-193.

43. The TIME-2b study: a study of AKB-9778, a novel tie 2 activator, in patients with non-proliferative diabetic retinopathy (NPDR) - tabular view - clinicalTrials.gov [Internet]. Available from: https://clinical trials.gov/ct2/show/record/NCT03197870. Accessed April 24, 2021.

44. Hussain RM, Neiweem AE, Kansara V, Harris A, Ciulla TA. Tie-2/ Angiopoietin pathway modulation as a therapeutic strategy for retinal disease. Expert Opin Investig Drugs. 2019;28(10):861-869. doi:10.1080/13543784.2019.1667333

45. Regeneron Pharmaceuticals. An open-label, dose-escalation study of the safety and tolerability of intravitreal (IVT) REGN910-3 and IVT REGN910 in patients with either neovascular AMD or DME [Internet]. clinicaltrials.gov; 2016. Available from: https://clinical trials.gov/ct2/show/NCT01997164. Accessed June 27, 2021.

46. Regeneron Pharmaceuticals. A randomized, double-masked, active-controlled, phase 2 study of the efficacy, safety, and tolerability of repeated doses of intravitreal REGN910-3 in patients with diabetic macular edema [Internet]. clinicaltrials.gov; 2018. Available from: https:// clinicaltrials.gov/ct2/show/NCT02712008. Accessed June 27, 2021.

47. Regeneron Pharmaceuticals. A randomized, double-masked, active-controlled phase 2 study of the efficacy, safety, and tolerability of repeated doses of intravitreal REGN910-3 in patients with neovascular age-related macular degeneration [Internet]. clinicaltrials. gov; 2019. Available from: https://clinicaltrials.gov/ct2/show/ NCT02713204. Accessed June 27, 2021.

48. Sato TN, Tozawa Y, Deutsch U, et al. Distinct roles of the receptor tyrosine kinases Tie-1 and Tie-2 in blood vessel formation. Nature. 1995;376(6535):70-74. doi:10.1038/376070a0

49. Regula JT, Lundh von Leithner P, Foxton R, et al. Targeting key angiogenic pathways with a bispecific CrossMAb optimized for neovascular eye diseases. EMBO Mol Med. 2017;9(7):985. doi:10.15252/ emmm.201707895

50. Gahn GM, Khanani AM. New therapies of neovascular AMD beyond anti-VEGF injections. Vis Basel Switz. 2018;2(1):15.

51. Chakravarthy U, Bailey C, Brown D, et al. Phase I trial of antivascular endothelial growth factor/anti-angiopoietin 2 bispecific antibody RG7716 for neovascular age-related macular degeneration. Ophthalmol Retina. 2017;1(6):474-485. doi:10.1016/j. oret.2017.03.003

52. Sahni J, Dugel PU, Patel SS, et al. Safety and efficacy of different doses and regimens of faricimab vs ranibizumab in neovascular age-related macular degeneration: the AVENUE phase 2 randomized clinical trial. JAMA Ophthalmol. 2020;138(9):955-963. doi:10.1001/ jamaophthalmol.2020.2685

53. Khanani AM, Patel SS, Ferrone PJ, et al. Efficacy of every four monthly and quarterly dosing of faricimab vs ranibizumab in neovascular age-related macular degeneration: the STAIRWAY phase 2 randomized clinical trial. JAMA Ophthalmol. 2020;138(9):964-972. doi:10.1001/jamaophthalmol.2020.2699

54. Sahni J, Patel SS, Dugel PU, et al. Simultaneous inhibition of angiopoietin-2 and vascular endothelial growth factor-A with faricimab in diabetic macular edema: BOULEVARD phase 2 randomized trial. Ophthalmology. 2019;126(8):1155-1170. doi:10.1016/j. ophtha.2019.03.023 
55. Vandekerckhove K ROA. Aflibercept versus ranibizumab for treating persistent diabetic macular oedema. Int Ophthalmol. 2015;35 (4):603-609. doi:10.1007/s10792-015-0081-7

56. Roche H-L. A phase III, multicenter, randomized, double-masked, active comparator-controlled study to evaluate the efficacy and safety of faricimab (RO6867461) in patients with diabetic macular edema (YOSEMITE) [Internet]. clinicaltrials.gov; 2021. Available from: https://clinicaltrials. gov/ct2/show/NCT03622580. Accessed April 22, 2021.

57. Roche H-L. A Phase III, multicenter, randomized, double-masked, active comparator-controlled study to evaluate the efficacy and safety of faricimab (RO6867461) in patients with diabetic macular edema (RHINE) [Internet]. clinicaltrials.gov; 2021. Available from: https:// clinicaltrials.gov/ct2/show/NCT03622593. Accessed April 22, 2021.

58. Roche H-L. A Phase III, multicenter, randomized, double-masked, active comparator-controlled study to evaluate the efficacy and safety of faricimab in patients with neovascular age-related macular degeneration (LUCERNE) [Internet]. clinicaltrials.gov; 2021. Available from: https:// clinicaltrials.gov/ct2/show/NCT03823300. Accessed April 22, 2021.
59. Roche H-L. A Phase III, multicenter, randomized, double-masked, active comparator-controlled study to evaluate the efficacy and safety of faricimab in patients with neovascular age-related macular degeneration (TENAYA) [Internet]. clinicaltrials.gov; 2021. Available from: https:// clinicaltrials.gov/ct2/show/NCT03823287. Accessed April 22, 2021.

60. Ingelheim B. Safety, tolerability and pharmacodynamics of single rising intravitreal and multiple rising intravitreal doses of BI 836880 in patients with wAMD (open label, non-randomized, uncontrolled). [Internet]. clinicaltrials.gov; 2021. Available from: https:// clinicaltrials.gov/ct2/show/NCT03861234. Accessed June 27, 2021.
Clinical Ophthalmology

\section{Publish your work in this journal}

Clinical Ophthalmology is an international, peer-reviewed journal covering all subspecialties within ophthalmology. Key topics include: Optometry; Visual science; Pharmacology and drug therapy in eye diseases; Basic Sciences; Primary and Secondary eye care; Patient Safety and Quality of Care Improvements. This journal is indexed on PubMed

Submit your manuscript here: https://www.dovepress.com/clinical-ophthalmology-journal
Dovepress

Central and CAS, and is the official journal of The Society of Clinical Ophthalmology (SCO). The manuscript management system is completely online and includes a very quick and fair peer-review system, which is all easy to use. Visit http://www.dovepress.com/ testimonials.php to read real quotes from published authors. 